\title{
Multimodal Imaging in Lung Cancer: It Is Time to Change
}

\section{Pablo Aguiar and Álvaro Ruibal*}

Nuclear Medicine Service, IDIS, University Hospital, Universidade de Santiago de Compostela, Spain

The imaging techniques can be classified into two main groups: Structural/morphological imaging (SMI), which includes $\mathrm{X}$ rays (XR), computed tomography (CT), ultrasounds (US) as well as some varieties of magnetic resonance (MRI), and shows anatomic-morphological aspects, and molecular imaging (MI), which includes nuclear medicine (SPECT, PET), fMRI, optical and nanosystems techniques, and provides information about biochemistry/biological activity, often before structural changes. According to Society of Nuclear Medicine and Molecular Imaging, MI "is the visualization, characterization, and measurement of biological processes at the molecular and cellular levels in humans and other living systems". MI procedures are noninvasive, safe and painless. Its sensitivity is greater than SMI, but it lacks anatomical detail, which has led to the development of multimodal imaging, combining structural and molecular techniques, widely used at present in daily practice. The pillars of MI are biochemistry/biology, instrumentation and software, and its cycle is the following: study of biology/biochemistry of a process, establishment/definition of specific targets, and development of tracers, preclinical imaging, histological validation and finally clinical imaging. This new concept led to the individualized diagnostic and treatment, being the patient the center of the medical activity. "As opposed to the doctor-centric, curative model of the past, the future is going to be patient-centric and proactive" said Dr. Zerhouni (NIH Medline Plus Winter 2007). The doctor must adapt to the needs of the own patient and this fact requires a true change of heart, because $\mathrm{MI}$ is intimately tied to the biology of the disease to analyzing. A new and strong interrelationship came into being: a bidirectional system biology-imaging that will allow to be much more effective in the daily practice, not only in relation to diagnosis (specific and early), but also with therapy (guide cancer treatment selection and evaluate early treatment response). There is an absolute necessity to lock the two together. Likewise, in the future the biology of a disease will indicate us what is the most adequate imaging technique and vice versa. In this regard, we know that in non-small cell lung cancer (NSCLC), ALK+ status is associated with distinct characteristics at CT imaging (CT radiogenomic characterization) [1], and that in lung adenocarcinomas 18F-FDG uptake values are related with expression levels of cellular Glucose Transporters and EGFR mutations. For this reason, different EGFR mutations correlate with different FDG uptake values.

Several genetic abnormalities specific in NSCLC and small cell lung cancer (SCLC) have been studied and some molecular pathways have been defined (EGFR family, Ras-Raf-Mek, PI3K-Akt-mTOR, LKb1, TITF1 (amplification of thyroid transcription factor 1) as targets of some imaging techniques and therapies [2]. Particular interest have micro-RNAs, a subgroup of emerging imaging biomarkers of primary and metastatic cancer, involved in gene expression, different stages of tumor evolution and with important clinical implications. They can be studied in tissues and biological fluids (plasma/serum) and some of them show an aberrant expression in the plasma levels (iR20a, miR-223, miR-21, miR-221 and miR-145) and can serve as noninvasive markers in the detection in early-stage NSCLC patients. Another, miRNA205 can be used to differentiate squamous cell carcinoma from adenocarcinoma of lung. miRNAs are also interesting because they can reduce the false positive rates obtained with different imaging techniques [3]. Other biomarkers with clinical usefulness include proteins, glycans, circulating cell-free DNA and several matrix metalloproteases, some of which (MMP13) have great interest in early pulmonary invasive adenocarcinoma. PET with different radiotracers is the primary modality in early predication of targeted therapy for NSCLC and some of them offer new opportunities in daily practice as $18 \mathrm{~F}$-fluoroazomycin arabinoside (FAZA), predictive parameter of treatment outcome in NSCLC patients, or $58 \mathrm{Ga}$-ventilation/perfusion $\mathrm{PET}$ in the context of radiation therapy.

Molecular imaging has evidenced a new feature of great importance: the intratumoral metabolic heterogeneity which seems to be related to histopathological features and outcome in NSCLC, showing the most beneficial areas for biopsies and playing an important role in planning for radiotherapy and in the follow-up of patients under chemotherapy $[4,5]$. MI will show us its effectiveness (total, partial or complete remission) and opens the possibility to do biopsies in tumor areas still metabolically actives after a treatment and to detect a residual tumor that could be biologically different to primary.

As mentioned above, MI is intimately related to the biology of the tumor and therefore both the diagnosis and treatment of multiple diseases could be individualized for each patient. In this regard, last advances in software have provided clinically feasible, quantitative multimodality images of tissue pathophysiology. Firstly, great efforts were focused on the integration of anatomical and functional characteristics, such as PET/CT, PET/MR, SPECT/CT and SPECT/MR and later many software applications have been implemented for the integration of multiple quantitative measurements in order to achieve a more-comprehensive characterization of the tumor phenotype [6]. Nowadays, the current multimodal images generate enormous amount of complementary quantitative data, which offer unique insights into opportunities to optimize care for individual patients. Nevertheless, obtaining such quantitative parameters from the images is not straightforward, and an important technical optimization and improved biological interpretation of the findings is still required. At present, this approach can already be applied informatively in clinical trials of cancer therapeutics using existing tools, but the findings should be carefully interpreted. In this regard, the great advances in the development of new scanners and in the search of specific imaging tracers have been accompanied by major changes in how to assess the image and extract clinically useful parameters. Thus, multiple advanced methods for image processing have been developed and they are currently being used for image interpretation in addition to the visual inspection. Although the qualitative evaluation based on visual assessment of the images is still an essential tool, and it may be suffice in many cases of evaluation of the response of different diseases, quantitative techniques for $\mathrm{MI}$ are increasingly being recognized as an essential tool for diagnosis, determination of prognosis, and response

*Corresponding author: Álvaro Ruibal, Nuclear Medicine Service, IDIS, University Hospital, Universidade de Santiago de Compostela, Spain, Tel: +34981950088; Fax: +34981950538; E-mail: pablo.aguiar.fernandez@sergas.es

Received September 08, 2015; Accepted September 10, 2015; Published September 17, 2015

Citation: Aguiar P, Ruibal Á (2015) Multimodal Imaging in Lung Cancer: It Is Time to Change. Lung Dis Treat 1: e101. doi:10.4172/2472-1018.1000e101

Copyright: $\odot 2015$ Aguiar $P$, et al. This is an open-access article distributed under the terms of the Creative Commons Attribution License, which permits unrestricted use, distribution, and reproduction in any medium, provided the original author and source are credited. 
monitoring in oncology. In particular, the evaluation of the response of solid tumors to therapy is challenging and requires some form of quantification in addition to the qualitative evaluation, which is discussed below.

The greatest advances have been carried out in PET studies with FDG, a glucose analog called fluorodeoxyglucose for the tissue uptake of glucose, which in turn is closely correlated with certain types of tissue metabolism. In this regard, various quantitative measures can be derived from the images, such as the rate of metabolism of glucose, which can be obtained by using a pharmacokinetic model to data from dynamic FDG-PET studies. Simplified quantitative measures obtained from static FDG-PET have been also proposed, such as the standardized uptake value (SUV), which is probably the most widely used method for the quantification of FDG-PET scans. It represents the glucose uptake within a tumor, measured over a certain interval.

In NSCLC, quantitative PET has been shown to provide prognostic information and multiple image-derived parameters such as metabolically active tumor volume (MATV), mean standardized uptake value ( $\mathrm{SUV}_{\text {mean }}$ ), and total lesion glycolysis (TLG) provide an accurate assessment of tumor burden with high prognostic value. Furthermore, intra-tumor heterogeneity has been associated with treatment failure and therefore its quantification is particularly interesting. Several methodologies can be used for the assessment of intra-tumor heterogeneity such as SUV coefficient of variation, area under the curve of the cumulative histogram, and textural-features analysis. On the other hand, hypoxia is also related to poor prognosis in NSCLC and it has great potential to individualize patient treatment. Hypoxia PET imaging can be carried out with different tracers, such as HX4 or FMISO. In these studies, the estimation of the tumor hypoxic fraction is required, which can be directly calculated based on a tumorto-background ratio [7].

Finally, it has to be mentioned that an important optimization of the imaging techniques and specially for improving biological interpretation of multimodality image-derived parameters are still required. Quantitative imaging can be affected by many technical and physiologic factors and several recommendations are currently available for improving the quantitative accuracy of biological parameters extracted from the images. These recommendations propose to keep in mind and work carefully acquisition protocols and data analysis [8] and using advanced tomographic reconstruction methods [9]. In addition, all quantification methods should be previously validated using preclinical imaging techniques, which allow us ex-vivo analysis aimed at testing the accuracy of the image-derived parameters.

\section{References}

1. Yamamoto S, Korn RL, Oklu R, Migdal C, Gotway MB, et al. (2014) ALK molecular phenotype in non-small cell lung cancer: $C T$ radiogenomic characterization. Radiology 272: 568-576.

2. Herbst RS, Heymach JV, Lippman SM (2008) Lung cancer. N Engl J Med 359 1367-1380.

3. Boeri M, Sestini S, Fortunato O, Verri C, Suatoni P, et al. (2015) Recent advances of microRNA-based molecular diagnostics to reduce false-positive lung cancer imaging. Expert Rev Mol Diagn 15: 801-813.

4. MacManus M, Everitt S, Hicks RJ (2015) The evolving role of molecular imaging in non-small cell lung cancer radiotherapy. Semin Radiat Oncol 25: 133-142.

5. McGranahan N, Swanton C (2015) Biological and therapeutic impact of intratumor heterogeneity in cancer evolution. Cancer Cell 27: 15-26.

6. Yankeelov TE, Abramson RG, Quarles CC (2014) Quantitative multimodality imaging in cancer research and therapy. Nat Rev Clin Oncol 11: 670-680.

7. Zegers CM, van Elmpt W, Reymen B, Even AJ, Troost EG, et al. (2014) In vivo quantification of hypoxic and metabolic status of NSCLC tumors using [18F] HX4 and [18F]FDG-PET/CT imaging. Clin Cancer Res 20: 6389-6397.

8. Boellaard R (2009) Standards for PET image acquisition and quantitative data analysis. J Nucl Med 50 Suppl 1: 11S-20S.

9. Aguiar P, Pino F, Silva-Rodríguez J, Pavía J, Ros D, et al. (2014) Analytical, experimental, and Monte Carlo system response matrix for pinhole SPECT reconstruction. Med Phys 41: 032501. 